\title{
Sirpenski Carpet Fractal QR code
}

\author{
Anupam Tiwari \\ Member (L-20108), Institute of Electronics and Telecommunication Engineers- $N$ Delhi
}

\begin{abstract}
QR (Quick Reaction) code is 2-dimension (2-D) mobile barcodes that are easily scanned using any modern mobile camera-equipped handsets/ phone to provide easy connectivity with internet. This QR Code will then be converted (called "dequrified") into a piece of (interactive) text and/or link. Presently QR codes are popular, because they are used for commercial tracking, logistics, inventory control, and advertising. In this paper two versions of Sierpinski Carpet are proposed as QR code. Sierpinski Carpet Fractal is well known Fractal and easy to generate. QR code, in Sierpenski pattern has main advantage of reduction of errors, easy generation, more symmetry, and enhanced data capacity. Sierpinski Carpet $Q R$, the position for ensign is also better with definite scale $\left(1 / 3^{\text {rd }}\right)$ space generated by first iteration for putting ensign.

Keywords: Sierpenski Carpet, QR, Error code.
\end{abstract}

\section{Introduction}

In present world people on move want to connect with internet with mobile devices. QR code is connect between mobile device containing camera with internet [1] .The basic QR code is a kind barcode with 2-D, of matrix symbol, which was developed by the Japanese company Denson-Wave in 1994. QR codes are used for Initiating a Web download, sending an SMS or email message, dialling a number, $\square$ consuming data such as business card information directly from the code. Figure-1 shows the basic structure of QR code. There are quiet zone, position detection patterns, separators for position detection patterns, timing patterns, alignment patterns, format information, version information, data, and error correction code words. The main features of QR code contain large capacity, small printout size, high speed scanning, advanced error correcting, and freedom direction scanning. The overall are summarized as follows.

- High data capacity: QR code can store 7,089 numeric characters and 4,296 alphanumeric characters, and 1,817 kanji characters.

- High speed scanning: Smart phone/mobile with camera can get the content from a QR quickly and easily.

- Small printout size: QR Codes carry data on both horizontally and vertically, so due to 2-D is better as 1D barcode.

- Advance error correcting: There is a 30\% tolerance for error within any QR Code. The codes still can be recognized correctly as it is $2-\mathrm{D}$.

- Freedom direction scanning: QR can be scanned from any direction.
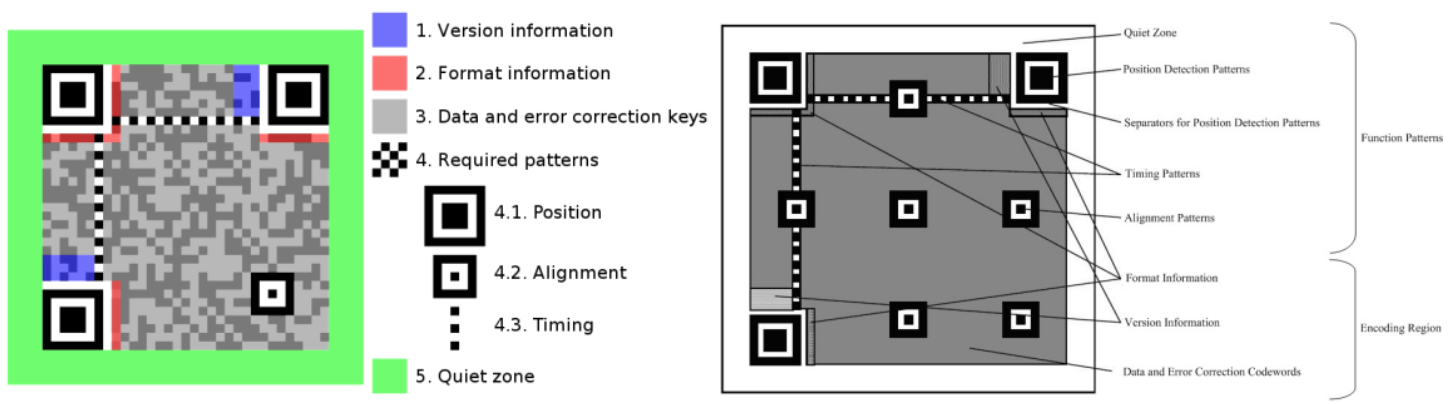

Figure-1

The QR code standard is fully described in the ISO/IEC 18004E International Standard and is available for purchase from the ISO Standard Organization for a nominal fee.

\section{Existing Patterns Of QR Code}

There are different patterns of Eye (square, diamond, etc), modules (Square, sieve, light roundness, etc) external eye colour, internal eye colour and background colour, foreground colours. Figure-2(Left) shows Diamond eye with foreground single colour blue. Shades of colour can also be used across length /breath or diagonal. Ensign can be also put at centre as shown in Figure -2 (Middle). 


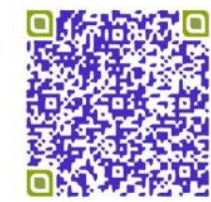

Figure-2(Left)

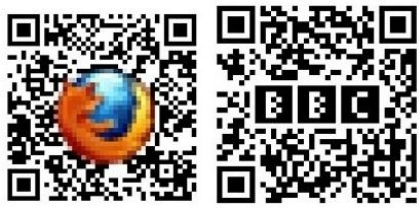

Figure-2(Middle)

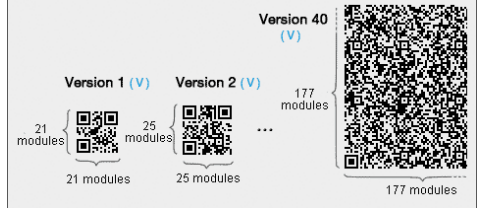

Figure-2(Right)

The standard specifies 40 versions (sizes) of the QR code from the smallest $21 \times 21$ up to $177 \times 177$ modules in size as shown in Figure-3(Right). The error level in QR code is as shown in the below table. The standard specifies four different principal schema, Numeric, Alphanumeric, Binary and Kanji as tabulated below.

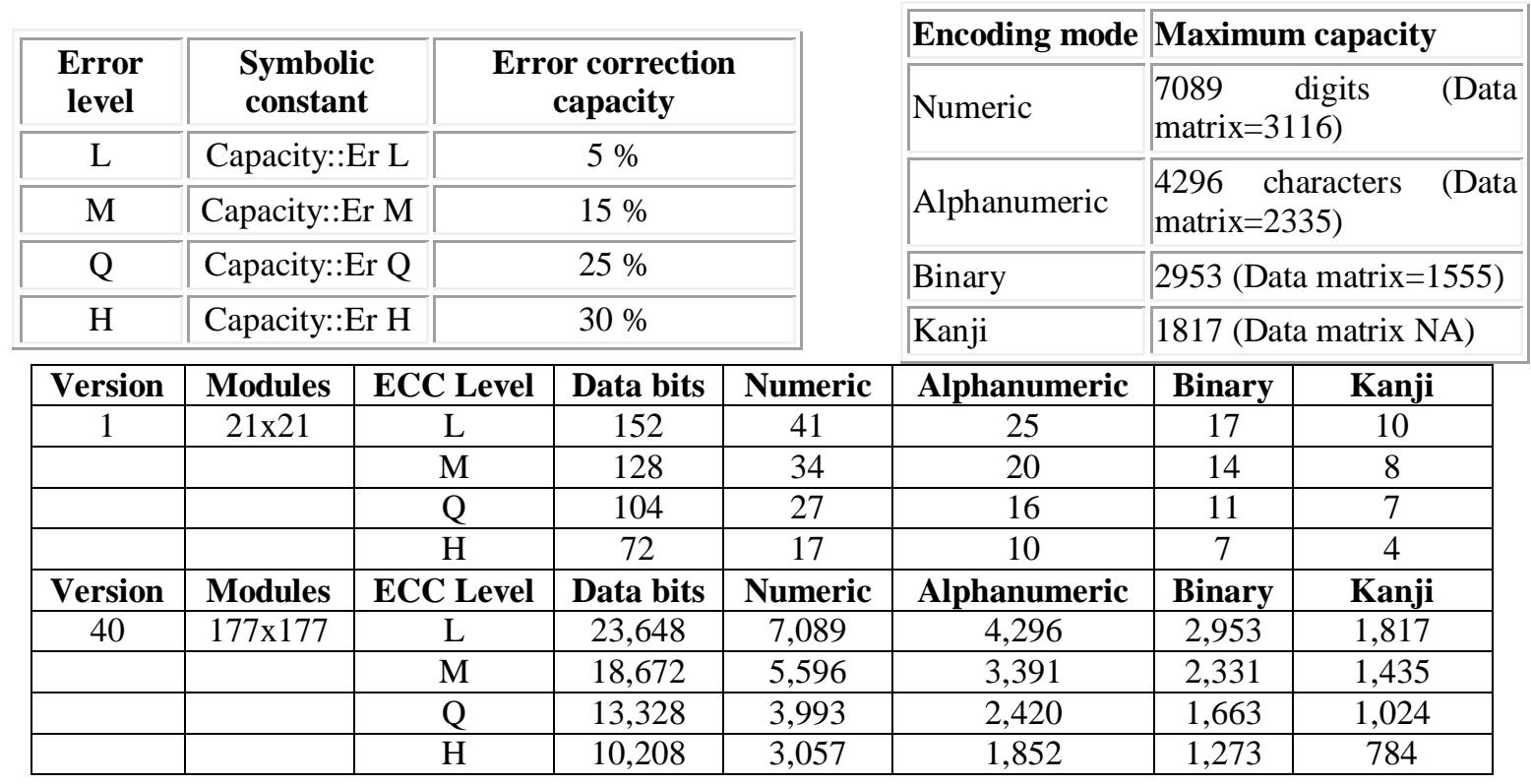

$W=(8 * 2+(4 V+1)) X+2 Q$

$\boldsymbol{W}=$ Width of QR code (including Quite zone), $\boldsymbol{H}=$ Height of QR code (including Quite zone), $\boldsymbol{X}=$ Width of $\mathrm{QR}$ Module, $Q=$ Width of the quite Zone; $Q=4 X, V$ is the version which is 1 to 40 .

Now, let us take, $\boldsymbol{V} \max =40, X \min =1, Q=4 X$.

$\boldsymbol{W} \max =(8 * 2+(4 * \mathbf{4 0}+1)) \boldsymbol{X}+2 \boldsymbol{Q}$

$=(177) X+2 * 4 X$

$=177 X+8 X$

$=185 X$

$=185$ pixel

\section{Sierpenski Carpet Fractal}

To construction of the Sierpinski Carpet Fractal begins with a square [2]. The square is cut into 9 congruent sub squares in a 3-by-3 grid, and the central sub square is removed this is first iteration. The same procedure is then applied recursively to the remaining 8 sub-squares, this process is infinite. The Hausdorff dimension of the carpet is $\log 8 / \log 3 \approx 1.8928$.

\section{Proposed QR Sierpenski Carpet}

There are two Sierpenski Carpet QR code is proposed. First (left) is third iteration Sierpenski Carpet, the eye of QR code is kept at centre and alignment are nine green squares (remove centre square is first iteration, which can be used for ensign). Figure -3 is Sierpenski Carpet QR code, with quiet zone (Red boundary), version (blue horizontal bar), Timing (Red Squares), data and error code. The eye is at centre and green eight squares are for alignment. In Second (right) Sierpinski Carpet with three yellow positioned with one red alignment square. White squares for timing and sync, quite zone is outside the carpet. Data with error code can be put for rest of the location of carpet. Sierpinski Carpet is easily generated with small computer code program. Mathematically it is proved that this form of Fractal is compact and data storage can be enhanced with less error. 

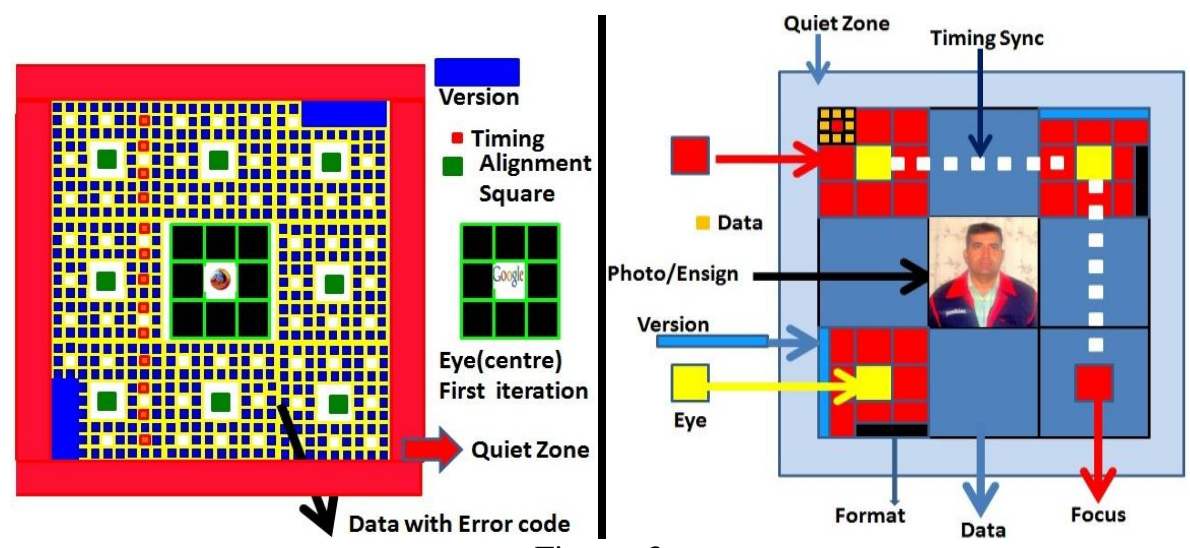

Figure -3

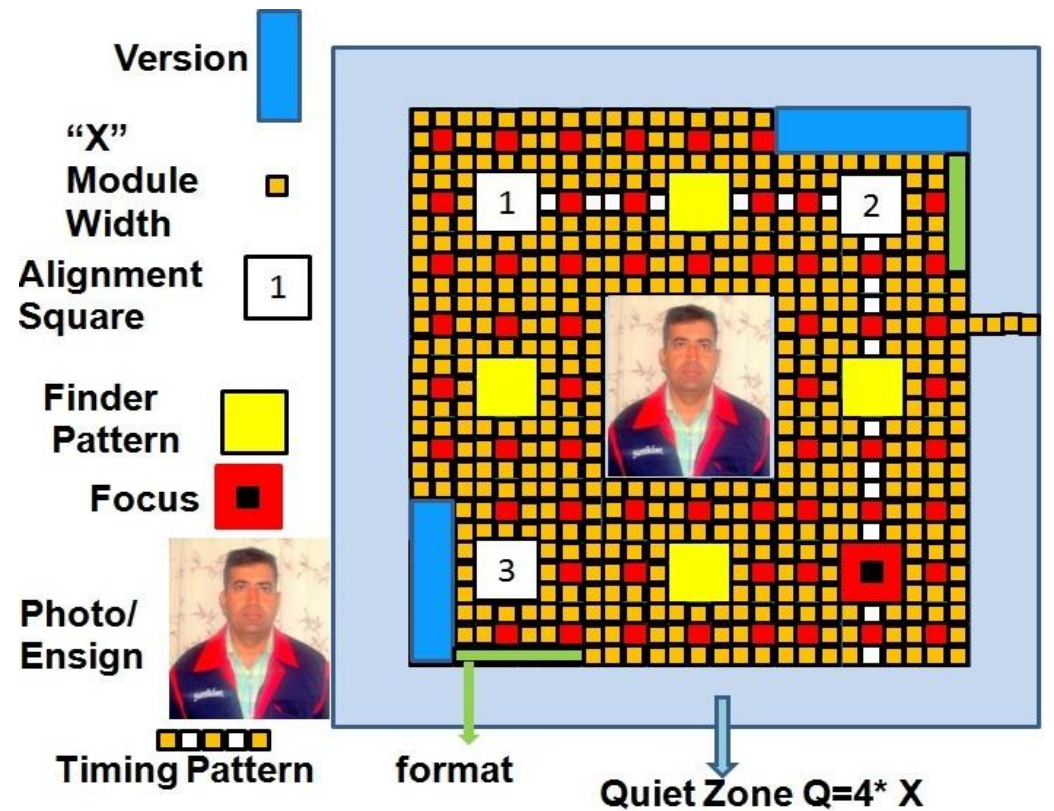

Figure -4 (Sierpinski Carpet QR Code with Ensign)

- With Sierpenski QR code, the main advantage of reduction of errors, easy generation, more symmetry and enhanced data capacity.

- $\quad$ Sierpinski QR is iterative so as per data iteration can be taken; Figure -4 is $3^{\text {rd }}$ Iteration Sierpinski Carpet in which alignment pattern is three squares marked as 1,2and 3 and the ratio is 2:3:2. Extra finder pattern is of yellow squares.

- $\quad$ Sierpinski QR is more beautiful with good aesthetic look. Synchronisation and timing data frame doesn't interfere with alignment pattern. Centre unused square can be utilised for logo, thus logo doesn't reduce data space.

- Version and Module as per data can be selected, if more data then "Seed" dimension can be increased as shown in Figure-5. As per data dimension of seed can be fixed.

- Format and masking can be put as per data and requirement of contrast. Sierpinski Carpet QR code is having better masking as compared to ordinary black and white QR code.

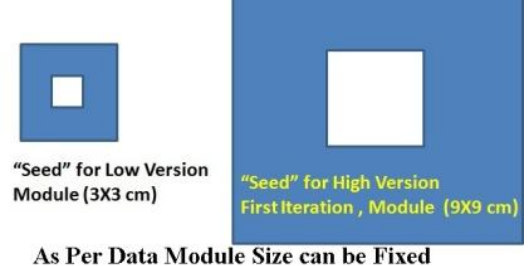

As Per Data Module Size can be Fixed

Figure -5 Versions and Module as per Data requirement 


\begin{tabular}{|l|l|l|c|}
\hline \multicolumn{1}{|c|}{ Version } & \multicolumn{1}{c|}{ Modules } & \multicolumn{1}{c|}{ ECC Level } & Data bits \\
\hline Low & $(3 \mathrm{cmX} 3 \mathrm{~cm})$ & L & As per iteration \\
\hline High & $(9 \mathrm{~cm} \mathrm{X} 9 \mathrm{~cm})$ & H & As per iteration \\
\hline
\end{tabular}

\section{Conclusion}

QR codes are presently growing their popularity because they connect mobile device with internet very easily. They are used for commercial tracking, logistics, inventory control, and advertising. Sierpinski Carpet Fractal QR code is proposed for QR codes as it is easy to generate by computer, Sierpinski Carpet is compact for data storage and unused center can be used for ensign. Depending on data size and iteration can vary, as iteration and size increases more data can be put with less error.

\section{Journal Papers:}

\section{References}

[1] K. Kiani, E. Kosari, and M. R. Simard, A New Method of Fractal Barcodes Identification, Proceedings of the World Congress on Books:

Engineering and Computer Science 2011 Vol I WCECS 2011, October 19-21, 2011, San Francisco, USA

[2] Heinz-otto Peitgen, D. S Chaos and fractals new frontiers of science (Springer-Verlag, 1992).

Bio data of The author

[3] Bio-data and e-mail is in QR code, (Please download free QR code from internet to decode bio-data), of Anupam Tiwari, or contact e-mail-anupamtiwari1972@rediffmail.com.
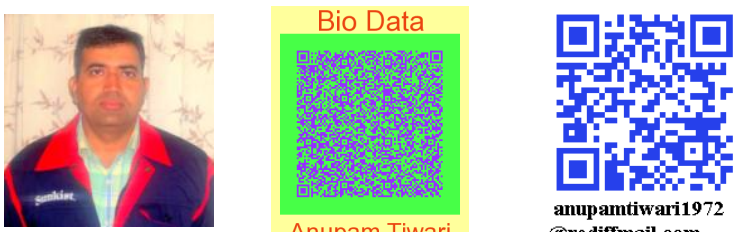

arediffmail.com 